\title{
Sub-nanometer resolution of an organic semiconductor crystal surface using friction force microscopy in water
}

\author{
Carlos Pimentel ${ }^{1,2}$, Shinto Varghese ${ }^{3}$, Seong-Jun Yoon ${ }^{4}$, Soo Young Park ${ }^{4}$, \\ Johannes Gierschner ${ }^{3}$, Enrico Gnecco $0^{3,5,6,7}$ and Carlos M Pina ${ }^{1,2,3}$ \\ ${ }^{1}$ Department of Crystallography and Mineralogy, Faculty of Geology, Complutense University of Madrid, c/ José Antonio Novais, 2, 28040 Madrid, Spain \\ 2 Instituto de Geociencias IGEO (UCM-CSIC), c/ José Antonio Novais, 2. E-28040. Madrid, Spain \\ ${ }^{3}$ Madrid Institute for Advanced Studies, IMDEA Nanoscience, c/ Faraday 9, Campus Cantoblanco,28049 Madrid, Spain \\ ${ }^{4}$ Center for Supramolecular Optoelectronic Materials and WCU Hybrid Materials Program, Department of Materials Science and Engineering, Seoul \\ National University, ENG 445, Seoul 151-744, Korea \\ ${ }^{5}$ Otto Schott Institute of Materials Research, Friedrich Schiller University Jena, Löbdergraben 32, 07743 Jena, Germany \\ ${ }^{6}$ Organic Semiconductor Optoelectronics, School of Physics and Astronomy, University of St Andrews, North Haugh, St. Andrews, KY16 9SS, Scotland
}

E-mail: enrico.gnecco@uni-jena.de

\begin{abstract}
Organic semiconductors (OSC) are attracting much interest for (opto)electronic applications, such as photovoltaics, LEDs, sensors or solid state lasers. In particular, crystals formed by small $\pi$-conjugated molecules have shown to be suitable for constructing OSC devices. However, the (opto)electronic properties are complex since they depend strongly on both the mutual orientation of molecules as well as the perfection of bulk crystal surfaces. Hence, there is an urgent need to control nano-topographic OSC features in real space. Here we show that friction force microscopy in water is a very suitable technique to image the free surface morphology of an OSC single crystal (TDDCS) with sub-nanometer resolution.
\end{abstract}

We demonstrate the power of the method by direct correlation to the structural information extracted from combined single crystal (SC-) and specular (s-) XRD studies, which allows us to identify the pinning centers encountered in the stick-slip motion of the probing tip with the topmost methyl groups on the TDDCS surface.

Keywords: friction force microscopy, high resolution, aqueous conditions, organic semiconductor crystals

(Some figures may appear in colour only in the online journal)

\section{Introduction}

With the rise of nanostructured solids, nanoscale imaging has become fundamental for material characterization. Despite the recent advances in scanning tunneling, atomic force and transmission electron microscopies (STM, AFM, TEM), there are a number of materials for which sub-nanometer resolu-tion images are still difficult to obtain.
This concerns espe-cially soft semiconducting (or insulating) materials, such as organic bulk crystals. Organic semiconductors have found broad interest for optoelectronic applications where detailed structural characterization is required to understand the complex interplay between structural factors and the (opto) electronic functionality [1-8]. 
This is especially evident for charge transport, where the conductivity of single- versus poly-crystalline materials can vary by orders of magnitude, since the grain boundaries in the polycrystalline samples can effectively diminish charge percolation $[7,8]$. Moreover, the photophysics are strongly influenced by morphological inhomogeneities, eventually leading to a breakdown of lumi-nescence efficiency in polycrystalline thin film samples due to exciton quenching at the interfaces $[5,6]$. Therefore, the detailed understanding of molecular orientations in thin films and crystals, particularly at interfaces (e.g. single crystal sur-faces, grain boundaries) is essential.

Structural imaging is commonly done by combining standard AFM with (polarized) absorption and/or emission spectroscopic techniques in the ultraviolet to infrared region [9]. Although the resolution of the latter techniques can be effectively brought below the optical diffraction limit, this is still a rather imprecise and indirect method to determine molecular orientations [10, 11]. Furthermore, the application of direct structural investigation of e.g. local grazing inci-dence $x$-ray diffraction (GI-XRD) is limited to rather large domain sizes [12]. For a comprehensive, although slightly dated, review on AFM combined to XRD the reader is referred to the work by Ward [14]. On the other hand, high-resolu-tion structural investigations with STM can only be done on very thin films of flat-lying organic conjugated molecules on conductive substrates [13]. Subnanometer resolution images of thin self-assembled organic films deposited on hard sur-faces have been reported using noncontact AFM in ultra-high vacuum (UHV) [15] and, more recently, using friction force microscopy (FFM) in water [16]. In this natural environment, capillary condensation between probing tip and surface is prevented. In this way single large molecules can be readily identified on complex systems such as membrane proteins [17] and, as first shown by Ohnesorge and Binnig [18], even atomic resolution is possible on bulk solid surfaces.

In the present work, we show that FFM in water is a noninvasive fast and cheap ex situ technique to resolve submolecular features of a soft semiconducting single crystal of a highly luminescent conjugated organic compound. In such materials, homogeneity of the crystal surface is of high importance, e.g. for single cavity lasing [16]. Although single molecules of organic crystals can be distinguished with FFM in ambient conditions [19], no sub-lattice resolution of bulk organic materials has been reported so far using this tech-nique. We will demonstrate the power of the method by direct correlation to the structural information extracted from com-bined single crystal (SC-) and specular (s-) XRD studies.

\section{Experimental}

The sample under investigation is 4,4'-dibutoxy-distyrylthio-phenebased crystal carrying cyano-groups in the vinylene units (TDDCS, see figures $1(A)$ and $1(B)$ ), which was syn-thesized as described in the supporting information (stacks.
A
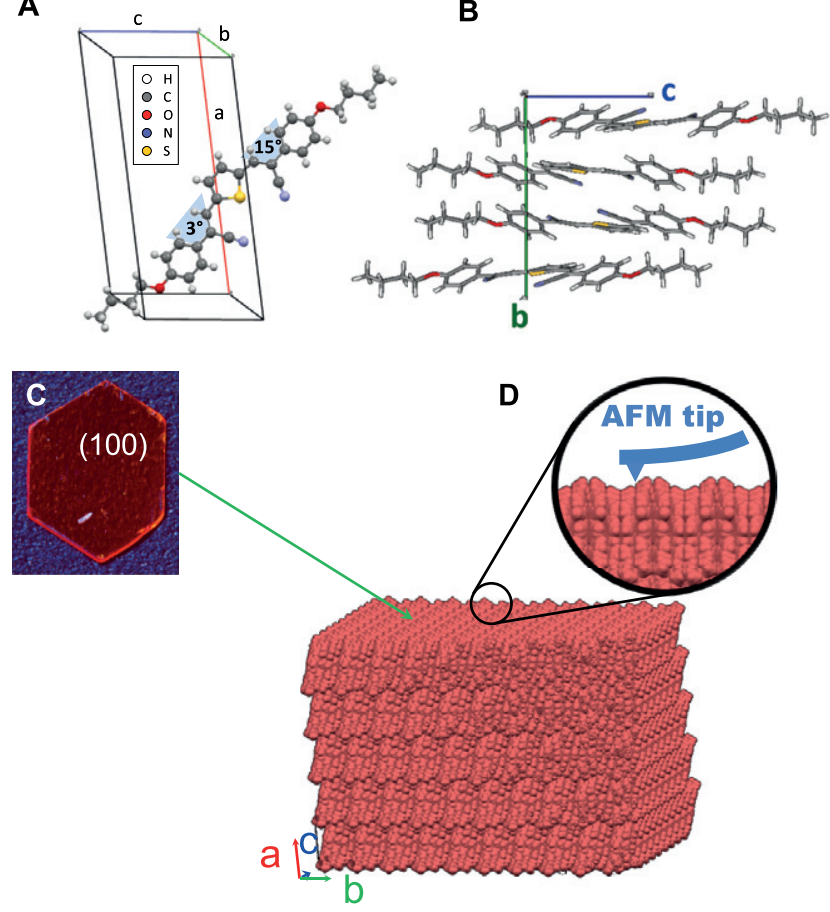

Figure 1. (A) Unit cell of a TDDCS crystal. Unit cell parameters: $a=1.99 \mathrm{~nm}, b=1.43 \mathrm{~nm}, c=0.90 \mathrm{~nm}, \alpha=90.0^{\circ}, \beta=98.6^{\circ}$, $y=90.0^{\circ}$. (B) Intermolecular arrangement. (C) Optical microscope image of the crystal investigated in our experiment. (D) Sketch of the AFM scanning on the $\left(\begin{array}{ll}1 & 0\end{array}\right)$ free surface of TDDCS.

iop.org/JPhysCM/28/134002/mmedia). The cyano-vinylene moieties have demonstrated to be suitable for targeted crystal design through secondary bonding interactions, with applica-tions in lasing, OLEDs and OFETs [4]. Single TDDCS crys-tals of some $\mathrm{mm}$ size and with a tabular habit (figure $1(\mathrm{C})$ ) were grown from solvent mixtures.

The single crystal structure was analyzed by using SMART-APEX II ULTRA (Bruker) in Central Instrument Facility, Gyeungsang National University. The file CCDC 977780 with the crystallographic data can be obtained free of charge via from the Cambridge Crystallographic Data Centre (www.ccdc.cam.ac.uk/ conts/retrieving.html). Details of the SC-XRD analysis are given in the SI. Specular (s-) XRD was measured using a Philips Xpert $\mathrm{x}$-ray diffractometer in

Bragg-Brentano geometry using $\mathrm{CrK} \alpha$ radiation (2.291 $\AA$ ) and a graphite monochromator on the secondary side.

The $\left(\begin{array}{lll}1 & 0 & 0\end{array}\right)$ face of the TDDCS crystal was studied using a commercial AFM (Multimode Illa Veeco Instruments) equipped with a closed fluid cell. This setup can only record up to three signals at the same time. The crystal was about $3 \mathrm{~mm}$ long, $1 \mathrm{~mm}$ wide and $0.1 \mathrm{~mm}$ thick and it was fixed on the AFM sample holder with double sided adhesive carbon

tape. To minimize tip-surface adhesion forces during AFM measurements, and take advantage of the insolubility of the crystal in water, the studied crystal was immersed in deion-ized water (Milli$Q$ Millipore; resistivity $18 \mathrm{M} \Omega \mathrm{cm}$ ) at room temperature. AFM images were taken in contact mode while displaying the height and friction signals. Only occasionally the AFM deflection signal was recorded. The images were 

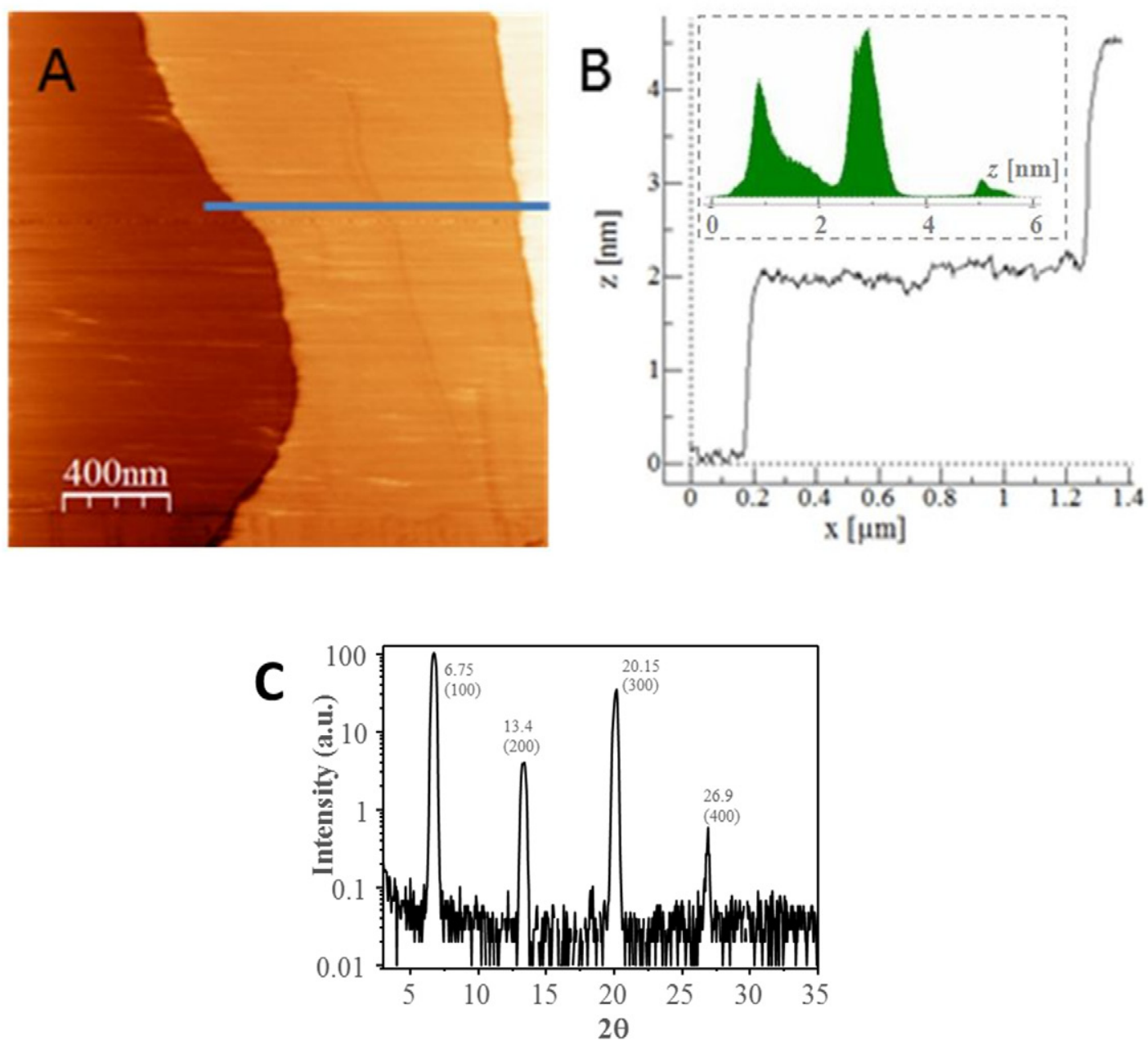

Figure 2. AFM analysis proving the flatness of the TDDCS $\left(\begin{array}{lll}1 & 0 & 0\end{array}\right)$ surface terraces in water. (A) Topography image; scan area: $2 \mu \mathrm{m} \times 2 \mu \mathrm{m}$. Scan velocity: $20 \mu \mathrm{m} \mathrm{s}^{-1}$; scan rate: $5 \mathrm{~Hz}$. (B) Surface profile corresponding to the horizontal blue line in (A). The histogram in the inset shows the statistical distribution of the surface height recorded in (A). (C) Specular XRD pattern of the plate-like facet of the TDDCS crystal.

collected at scan rates varying from 5 to $61 \mathrm{~Hz}$ and recording 512 lines per scan. A total number of about 450 AFM images were recorded. Sharp silicon nitride tips with a nominal radius of $2 \mathrm{~nm}$, and a triangular cantilever with a spring constant $k_{N}=0.06 \mathrm{~N} \mathrm{~m}^{-1}$, and a high level of uncertainty between 0.03 and $0.12 \mathrm{~N} \mathrm{~m}^{-1}$ (Bruker SNL-10), were used as pro-vided by the manufacturer. AFM data were analysed using the Nanoscope (5.30 $3 \mathrm{sr} 3$ ) and Nanotec (WSxM.4) software [20]. Measurements of friction forces were conducted using the

calibration methods proposed by Noy et al [21] and Lüthi et al [22]. Specifically, the lateral spring constant $k_{L}$ of the cantilever is obtained as

$$
k_{L}=\frac{2}{6 \cos ^{2} \theta+3(1+\nu) \sin ^{2} \theta}\left(\frac{L}{H}\right)^{2} k_{N},
$$

where $\theta=60^{\circ}$ is the base angle between the cantilever arms, $\nu=0.24$ is the Poisson's ratio of silicon nitride, $L=205 \mu \mathrm{m}$ is the length of the cantilever beam, and $H=5 \mu \mathrm{m}$ is the tip length. With the values provided by the manufacturer, $k_{L}=47 \mathrm{~N} \mathrm{~m}^{-1}$ (with a level of uncertainty between 23 and $94 \mathrm{~N} \mathrm{~m}^{-1}$ ). The lateral force values are finally obtained from the transverse signal measured by the four-quadrant photodiode using the conversion ratio (in $\mathrm{nN} / \mathrm{V}$ ) of the normal force multiplied by $3 H k \quad\left(/ 2_{\mathrm{LN}} \mathrm{LK}\right)$.

\section{Results and discussion}

SC-XRD analysis reveals that TDDCS crystallizes in a mono-clinic system $\left(\mathrm{SG}=P 2_{1} / c\right)$ with four molecules per unit cell and lattice parameters as given in the caption of figure 1. The slightly tilted molecules are oriented with their long axes in a unidirectional way, inclined against the $\left(\begin{array}{lll}1 & 0 & 0\end{array}\right)$ plane by about $5^{\circ}$. The exposed surface is found to correspond to the $\left(\begin{array}{lll}1 & 0 & 0\end{array}\right)$ plane, which according to the s-XRD data has an interplanar spacing of $55^{\circ}$. The exposed surface is found to correspond to the $\left(\begin{array}{ll}100 & 0\end{array}\right)$ plane, which according to the s-XRD data has an interplanar spacing of d100 = $1.97 \mathrm{~nm}$ (figure 2(C)), consistent with our AFM observation of monosteps of about $2.00 \mathrm{~nm}$ in height (fig-ures 2(A) and (B)). Note that the $\left(\begin{array}{lll}1 & 0 & 0\end{array}\right)$ surface of the TDDCS crystal is quite fragile since it is structurally defined by flexible alkyl chains, and indeed it is easily damaged if the feedback does not react fast enough when crossing the step edges. For this reason the scan rate was kept relatively slow $(5 \mathrm{~Hz})$.

High resolution FFM images of the terraces immersed in water provide detailed structural information of the TDDCS $\left(\begin{array}{lll}1 & 0 & 0\end{array}\right)$ surface. The friction map in figure 3 reveals a periodic 

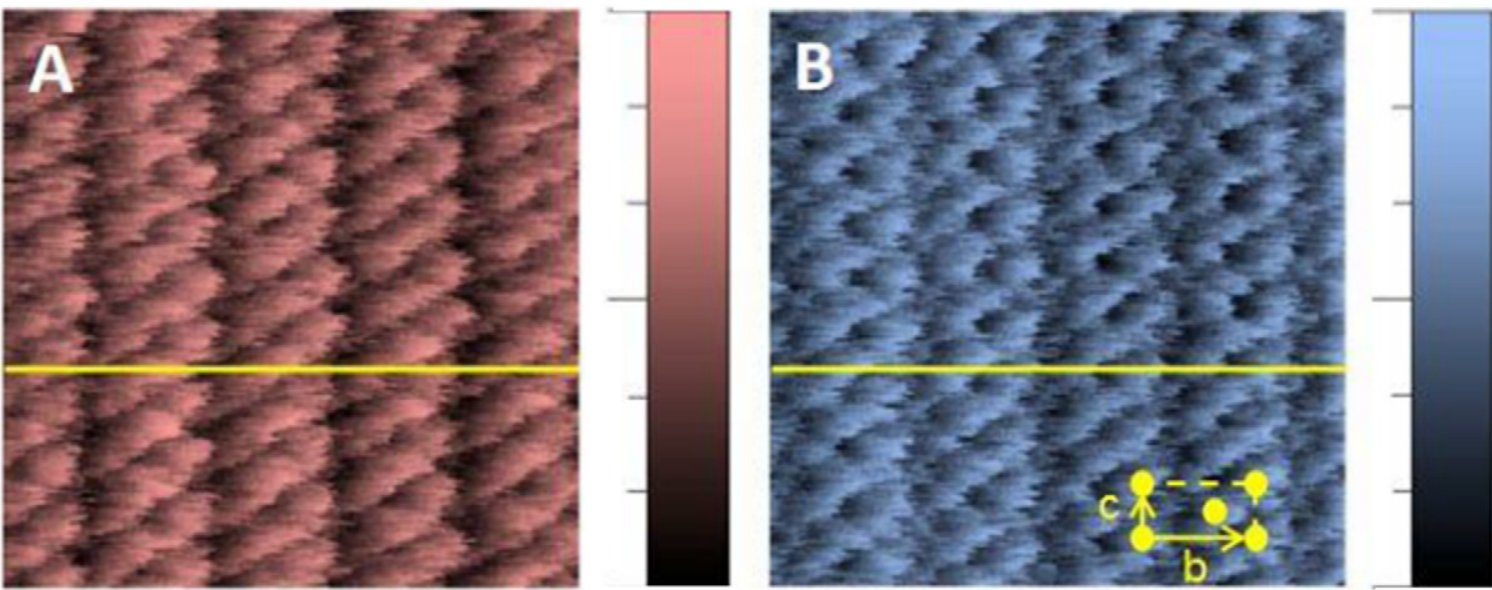

$-3 \mathrm{nN}$

$-8 \mathrm{nN}$
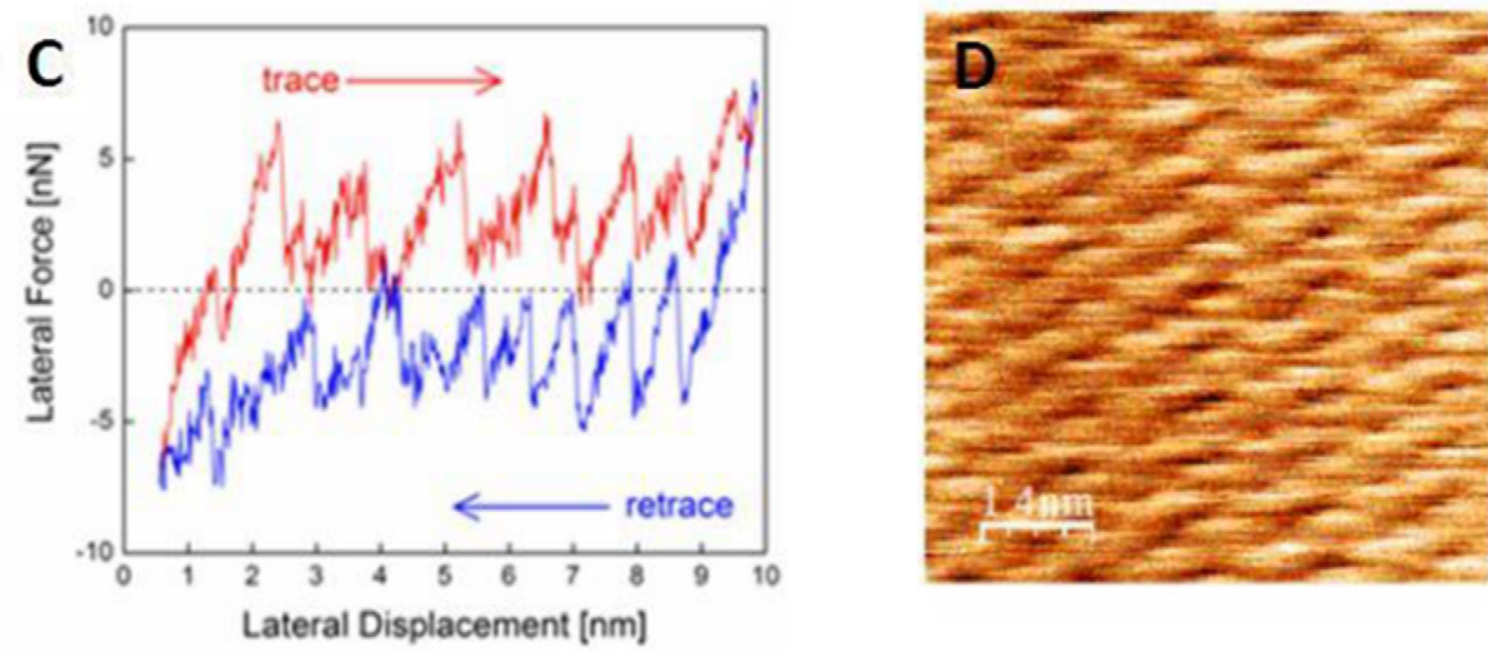

Figure 3. High-resolution FFM images $(7.2 \mathrm{~nm} \times 7.2 \mathrm{~nm}$ ) of TDDCS acquired while scanning (A) left to right and (B) right to left with a normal force of $4.2 \mathrm{nN}$ and a scan velocity of $1.2 \mu \mathrm{m} \mathrm{s}^{-1}$ (and a scan rate of $61 \mathrm{~Hz}$, considering the overscan). The average friction force is $2.3 \mathrm{nN}$. (C) Lateral force section corresponding to the yellow lines in (A) and (B). The yellow circles correspond to the topmost methyl groups, where the tip appears to be pinned in its stick-slip motion on the crystal surface. (D) Topography image corresponding to (A).

pattern, which can be directly correlated with rows of mol-ecules running parallel to the $c$ direction. In fact, a rectangular surface unit cell is clearly defined by peaks of friction with $b \sim 1.43 \mathrm{~nm}$ and $c \sim 0.91 \mathrm{~nm}$, in very good agreement with the nominal values $b=1.43 \mathrm{~nm}$ and $c=0.90$ nmobtainedby SC-XRD (figure $1(\mathrm{~A})$ ). The protruding groups have a

characteristic zig-zag arrangement with an angle of $117^{\circ}$, in agreement with our FFM images. The crystal surface was scanned over an area of $7.2 \mathrm{~nm} \times$

$7.2 \mathrm{~nm}$, showing a perfect periodic order at the molecular scale in the entire region. It is remarkable that such a resolution could not be achieved in air $(R H \approx 40 \%)$, where the TDDCS surface was irrevers-ibly damaged when imaged with comparable normal force values. This is possibly due to the absence of capillary forces in water leading to a strong reduction of adhesion between tip and surface and no damage of the TDDCS surface during scanning. Note that, compared to the previous image on much larger scale, the scan rate is quite high in figure $3(61 \mathrm{~Hz})$.
In this way the effect of thermal drift was reduced and the lateral force images become more stable.

The high contrast in FFM maps (as compared to the standard topography, see figure $3(\mathrm{D})$ ) is caused by the stick-slip motion of the probing tip. The elastic cantilever sup-porting the tip undergoes a periodic torsion while scanning the sample surface. This torsion is caused by the lateral force sensed by the tip, which is ultimately determined by the

lateral contact stiffness $k$ lat and the tip-sample interaction potential $U_{\text {int }}$ [23]. When the torsion angle reaches a critical value, depending on the corrugation of $U_{\text {int }}$, the tip suddenly slips into a new pinning site on the crystal lattice. Although the stick-slip mechanism is affected by thermal vibrations in the contact area and by collisions with water molecules, the slip events are clearly distinguished in the saw tooth profile of the lateral force signal (figure 3(C)), where they correspond to the vertical lines. From a comparison of figures $3(\mathrm{~A})$ and $(\mathrm{B})$ with the crystal structure of the TDDCS $\left(\begin{array}{lll}1 & 0 & 0\end{array}\right)$ surface (figure 4), 


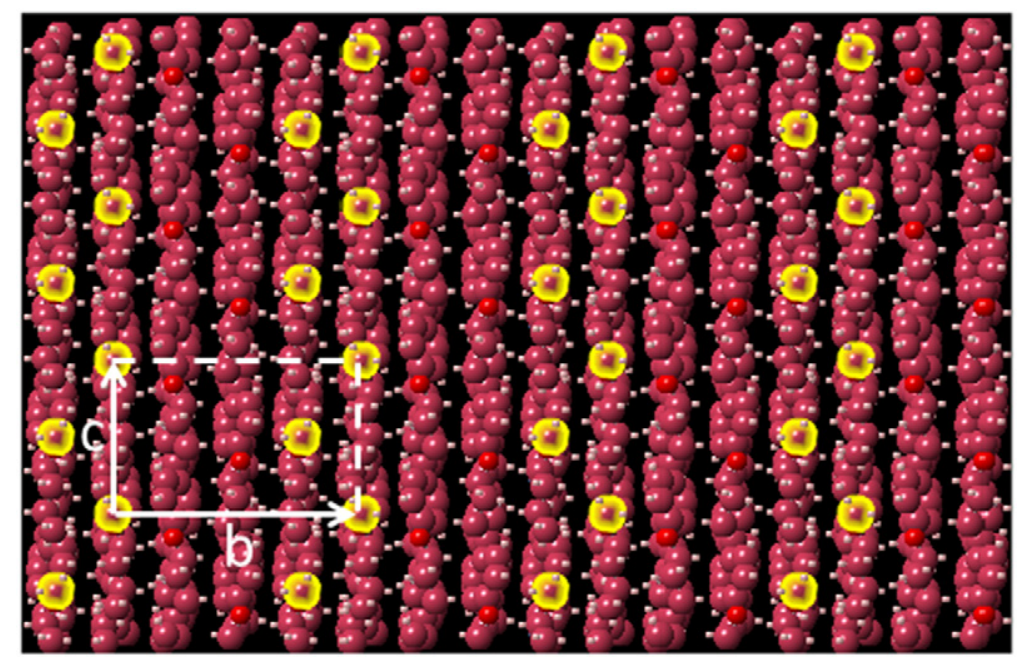

Figure 4. Atomistic model of TDDCS, projected on the (100) plane; yellow circles correspond to the topmost methyl groups (two per unit cells).
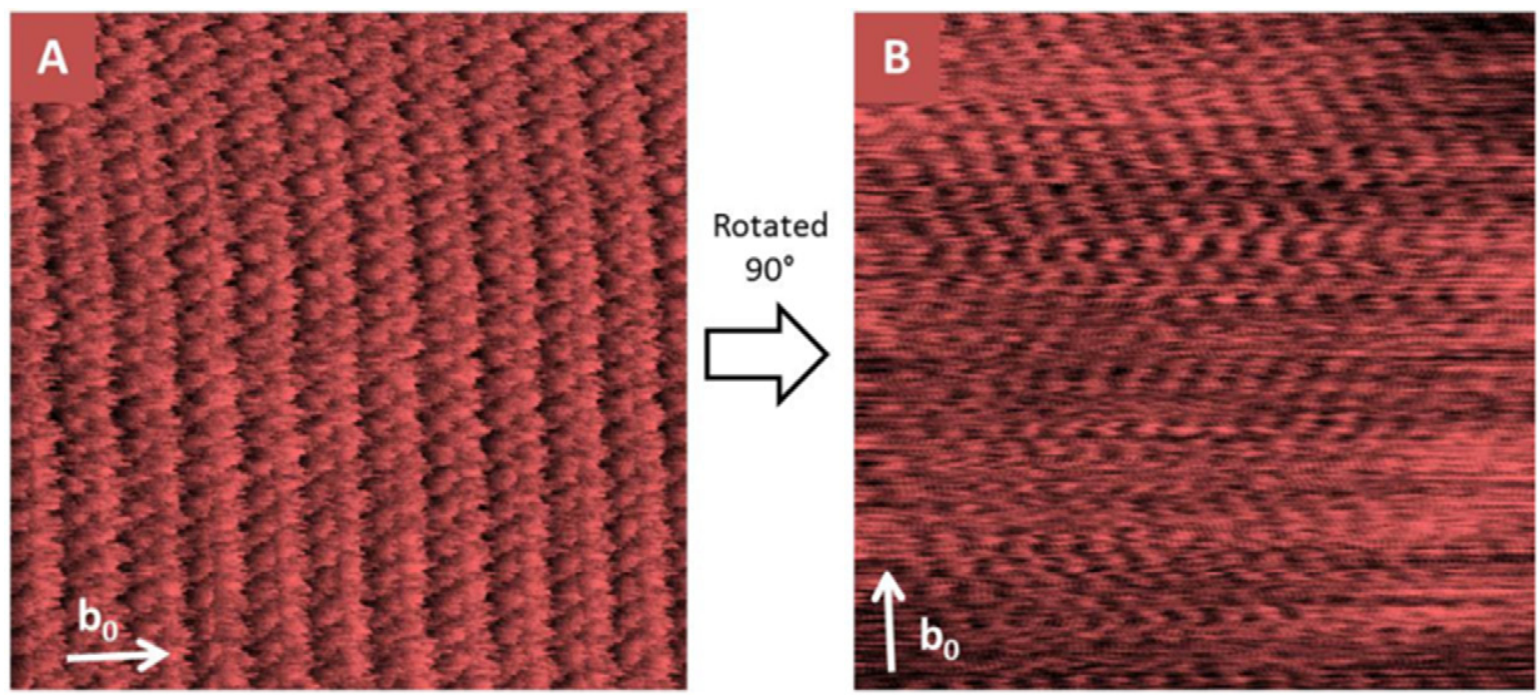

Figure 5. Friction anisotropy on the $\left(\begin{array}{lll}1 & 0 & 0\end{array}\right)$ face of TDDCS (scan areas: $17.3 \mathrm{~nm} \times 17.3 \mathrm{~nm}$ ): The contrast is quite different when the fast scan direction is (A) almost parallel to the $\mathrm{b}$ direction and (B) almost perpendicular to the b direction. Scan velocity: $2.4 \mu \mathrm{m} \mathrm{s}^{-1}$; scan rate: $61 \mathrm{~Hz}$.

we attribute the observed pinning sites to the terminal methyl groups. Note that the imaging mechanism is quite different from tapping mode AFM, where the tip is gently touching the sample while oscillating over it, without pulling the contact region aside. Compared to tapping AFM, the problems caused by spurious resonance peaks, which are frequently reported in liquid environments [24], are avoided using FFM.

From the average value of the lateral force peaks we esti-mate a static friction value $F_{\max }=5.9 \mathrm{nN}$. From the average value of the slope of the force versus (horizontal) distance curves in figure $3(\mathrm{C})$, we estimate an effective stiffness value $k_{\exp }=6.8 \mathrm{~N} \mathrm{~m}^{-1}$. According to [25] these values correspond to a 'friction parameter'

$$
\eta \pi_{\mathrm{ma}^{2}}{ }^{2} \quad F \quad k=\mathrm{x} \quad \text { e } \quad \mathrm{xp}(\mathrm{b} / 2) 1=6.6,
$$

where the value $b / 2=0.71 \mathrm{~nm}$ has been used for the average repetition distance in the curves. Note that, even if it is not apparent from the previous formula, $\eta$ is always positive, as seen from the definition which is also given in [25]. At this point the amplitude of the interaction potential is easily determined as
$U_{0}=(b / 2) F_{\max } / \pi=1.34 \times 10^{-18} \mathrm{~J}=8.3 \mathrm{eV}$. The lateral contact stiffness [25] $k=(1+1 / \eta) k_{\exp }=7.8 \mathrm{~N} \mathrm{~m}^{-1}$ turns out to be slightly larger than the slope $k_{\exp }$. The shear stress $\tau$ can be also estimated as the ratio between the maximum lateral force $F_{\max }=5.9 \mathrm{nN}$ and the size of the contact area, $A_{\text {con }}$. As usual in AFM, the last quantity can be quantified only roughly. Assuming that $A_{\text {con }}$ is in the order of $(b / 2) c=0.64 \mathrm{~nm}^{2}$ we end up with $\tau=F_{\max } /(b c / 2) \sim 10 \mathrm{GPa}$ (considering the large uncertainty in $F_{\max }$ only the order of magnitude of this quantity can be estimated). These values are common in FFM measurements on inorganic crystal surfaces [26], but we are not aware of similar estimations on organic semiconductors.

As a final remark, we note that the TDDCS surface is imaged in a different way if the sample is manually rotated by $90^{\circ}$ without modifying the orientation of the cantilever with respect to the scan direction (figure 5). Indeed the protruding molecular rows become hardly distinguishable when scanning along the $c$ direction of the surface. We observed a similar 
effect in friction force maps of a dolomite (104) surface using the same AFM setup in water [27]. Also in that case the topmost molecular groups (carbonate) acting as pinning centres for the stick-slip motion were disposed in a zigzag arrangement, which was better resolved while sliding along one of the two main crystallographic directions of the surface.

The reasoning in [27] can be partially repeated here. To this end, we focus on the unit cells with a 'basis' of two spots corresponding to the topmost methyl groups, already shown is figure 4. From this figure, it should be clear that a perfectly symmetric tip, if scanned along the $c$ direction, would spend the same time pinned on each of the two spots. However, the same tip would remain longer in the corner spots, when the scanning along the $b$ direction (left to right). According to the discussion in [27], this geometric effect only would lead to better contrast along $b$, as actually observed in a similar situation on a dolomite crystal. However, this is not the case in our measurements on TDDCS. A possible reason for that is the complication given by the assembly of the organic chains in parallel rows. Figure 4 suggests that the chains are more flexible, and the surface is more compliant, when the sample is sheared along the $b$ direction. A reduced lateral stiffness $k_{\text {exp }}$, according to the definition of the friction parameter, $\eta=4 \pi U_{0} /\left(k_{\mathrm{exp}} a^{2}\right)\left(U_{0}\right.$ is again the amplitude of the tip-surface interaction potential, whereas $a$ is now the generic repetition distance of the stick-slip) would lead to higher values of $\eta$ and enhanced contrast in the friction maps. However, we should also note that the repetition distance is larger along the $b$ direction, which would lead to a decrease of $\eta$. Altogether, the results in figure 5 lead us to conclude that the elastic effect prevails on the geometric one, but, in order to quantitatively substantiate this hypothesis, complex molecular dynamics simulations are needed which go well beyond the goal of this work. Here we only would like to add that, as a follow-up of our experimental observations, friction anisotropy could be used to distinguish between crystal domains with different molecular orientations, as suggested by previous FFM investigations, with much lower resolution, on lipid monolayers [28]. This is extremely interesting for the detailed structural characterization of polycrystalline samples, a subject of large interest in organic optoelectronic device structures [9].

\section{Conclusion}

To summarize, we have demonstrated how a peculiar structural characterization of soft molecular crystal surfaces with sub-molecular resolution can be achieved using FFM in liquid environment. We recognize in the saw-tooth profile of the lateral force the key factor for high resolution. Whenever a slip occurs, it can be easily discerned in the friction force maps. The only requisite is that, during the slip, the tip oscillations get quickly damped, which was the case up to the maximum speed that we applied (about $1 \mu \mathrm{m} \mathrm{s}^{-1}$ ). In this way we could estimate a shear strength between the tip and the crystal surface in the order of $10 \mathrm{GPa}$. The strong anisotropic effects observed in the friction maps also open an interesting pathway for the detection of molecular orientations in different domains, which in all recommends this application as a possible structural screening method for organic optoelectronics device structures. Additionally, we have shown that it is possible to obtain images with sub-molecular resolution just after setting up the microscope (less than $10 \mathrm{~min}$ ). This reduction in the time for image acquisition, together with the fact that UHV is not required, makes FFM in water a low cost and low time consuming surface characterization technique. To substantiate this conclusion a series of measurements at different scan rates should be performed. Only in this way one could exclude a negative influence of mechanical resonances and non-linearity of the scanner, which was not apparent in our measurements. Hydrodynamic effects may also influence the image stability at high scan speed.

\section{Acknowledgments}

The work at IMDEA and UCM was supported by the Spanish Ministerio de Economía y Competitividad (MINECO; projects CTQ2011-27317, MAT2012-34487, CTQ2014-58801), by the Comunidad de Madrid (project S2009/PPQ-1533), by the European Commission (Amarout II, FP7-PEOPLE2011-COFUND-291803), and performed in the context of the European COST Actions MP1302 (Nanospectroscopy) and MP1303 (Nanofriction). The work at Seoul National University was supported by the National Research Foundation of Korea (NRF) through a grant funded by the Korean government (MSIP; No 2009-0081571). CP is grateful to Spanish Ministry of Education, Culture and Sports for a FPU grant. AFM observations were carried out at ICTS Centro Nacional de Microscopía Electrónica at the Complutense University of Madrid. We also thank Jorge Moreno for technical assistance.

\section{References}

[1] Forrest S R and Thompson M E 2007 Organic electronics and optoelectronics Chem. Rev. 107 923-1386

[2] Miller R D and Chandross E A 2010 Materials for electronics Chem. Rev. 110 1-574

[3] Bredas J-L and Durrant J R 2009 Organic photovoltaics Acc. Chem. Res. 42 1689-857

[4] Varghese S and Das S 2011 Role of molecular packing in determining solid-state optical properties of $\pi$-conjugated materials J. Phys. Chem. Lett. 2 863-73

[5] Gierschner J, Lüer L, Milián-Medina B, Oelkrug D and Egelhaaf H-J 2013 Highly emissive H-aggregates or aggregation-induced emission quenching? The photophysics of all-trans para-distyrylbenzene. J. Phys. Chem. Lett. 4 2686-97

[6] Gierschner J and Park S Y 2013 Luminescent distyrylbenzenes: tailoring molecular structure and crystalline morphology J. Mater. Chem. C 1 5818-32

[7] Knipp D, Street R A, Volkel A and Ho J 2003 Pentacene thin film transistors on inorganic dielectrics: Morphology, structural properties, and electronic transport J. Appl. Phys. $93347-55$

[8] Horowitz G and Hajlaoui M E 2000 Mobility in polycrystalline oligothiophene field-effect transistors dependent on grain size Adv. Mater. 12 1046-50

[9] McNeill C 2011 Imaging the domain structure of organic semiconductor films. J. Polym. Sci. B 49 909-19 
[10] Zhang D, Heinemeyer U, Stanciu C, Sackrow M, Braun K, Hennemann L E, Wang X, Scholz R, Schreiber F and Meixner A J 2010 Nanoscale spectroscopic imaging of organic semiconductor films by plasmon-polariton coupling Phys. Rev. Lett. 104056601

[11] Loi M A, da Como E, Dinelli F, Murgia M, Zamboni R, Biscarini F and Muccini M 2005 Supramolecular organization in ultra-thin films of $\alpha$-sexithiophene on silicon dioxide Nat. Mater. 4 81-5

[12] Tersigni A, Qin X R, Kim C-Y, Gordon R A and Jiang D T 2011 Reciprocal-space mapping of lateral single-crystal domains with grazing-incidence $\mathrm{x}$-ray diffraction for tetracene on H/Si(00 1) Phys. Rev. B 84035303

[13] Barth J V, Costantini G and Kern K 2005 Engineering atomic and molecular nanostructures at surfaces Nature 437 671-9

[14] Ward M D 2001 Bulk crystals to surfaces: combining x-ray diffraction and atomic force microscopy to probe the structure and formation of crystal interfaces Chem. Rev. 101 1697-725

[15] Maier S, Fendt L-A, Zimmerli L, Glatzel T, Pfeiffer O, Diederich F and Meyer E 2008 Nanoscale engineering of molecular porphyrin wires on insulating surfaces Small $41115-8$

[16] Nita P, Pimentel C, Luo F, Milián-Medina B, Gierschner J, Pina C M and Gnecco E 2014 Molecular resolution friction microscopy of $\mathrm{Cu}$ phthalocyanine thin films on dolomite (104) in water Nanoscale 6 8334-9

[17] Bippes C A and Müller D 2011 High-resolution atomic force microscopy and spectroscopy of native membrane proteins Rep. Prog. Phys. 74086601

[18] Ohnesorge F and Binnig G 1993 True atomic resolution by atomic force microscopy through repulsive and attractive forces Science $2601451-6$

[19] Yamao T, Okuda Y, Makino Y and Hotta S 2011 Dispersion of the refractive indices of thiophene/phenylene co-oligomer single crystals J. Appl. Phys. 110053113
[20] Horcas I, Fernández R, Gómez-Rodríguez J M, Colchero J, Gómez-Herrero J and Baró A M 2007 WSXM: a software for scanning probe microscopy and a tool for nanotechnology Rev. Sci. Instrum. 78013705

[21] Noy A, Frisbie C D, Rozsnyai L F, Wrighton M S and Lieber C M 1995 Chemical force microscopy: exploiting chemically-modified tips to quantify adhesion, friction, and functional group distributions in molecular assemblies J. Am. Chem. Soc. 117 7943-51

[22] Lüthi R, Meyer E, Haefke H, Howald L, Gutmannsbauer W, Guggisberg M, Bammerlin M and Güntherodt H-J 1995 Nanotribology: an UHV-SFM study on thin films of C60 and $\mathrm{AgBr}$ Surf. Sci. 338 247-60

[23] Fessler G, Zimmermann I, Glatzel T, Gnecco E, Steiner P, Roth R, Keene T D, Liu S-X, Decurtins S and Meyer E 2011 Orientation dependent molecular friction on organic layer compound crystals Appl. Phys. Lett. 98083119

[24] Putman C A J., Van der Werf K O, De Grooth B G, Van Hulst N F and Greve J 1994 Tapping mode atomic force microscopy in liquid Appl. Phys. Lett. 64 2454-6

[25] Socoliuc A, Bennewitz R, Gnecco E and Meyer E 2004 Transition from stick-slip to continuous sliding in atomic friction: entering a new regime of ultra-low friction Phys. Rev. Lett. 92134301

[26] Meyer E, Gyalog T and Overney R 1999 Nanoscience: Friction and Rheology on the Nanometer Scale (Singapore: World Scientific)

[27] Pina C M, Miranda R and Gnecco E 2012 Anisotropic surface coupling while sliding on dolomite and calcite crystals Phys. Rev. B 85073402

[28] Liley M, Gourdon D, Stamou D, Meseth U, Fischer T M, Lautz C, Stahlberg H, Vogel H, Burnham N A and Duschl C 1998 Friction anisotropy and asymmetry of a compliant monolayer induced by a small molecular tilt Science $280273-5$ 